\title{
COMPATIBLE BIOMATERIALS USED IN RHINOLOGY SURGERY - OUR EXPERTISE
}

\author{
V. BUDU ${ }^{1,2}$, A. PANFILOIU ${ }^{1}$, ALEXANDRA GULIGA ${ }^{1}$, TATIANA DECUSEARA ${ }^{1}$, ANDREEA- \\ NICOLETA COSTACHE ${ }^{1}$, DIANAMARIA MISTRA ${ }^{3}$, LAVINIA SAVA $^{1}$, I. A. BULESCU ${ }^{2}, \mathrm{M}$. \\ TUSALIU ${ }^{1,2}$
}

${ }^{1}$ Institute of Phonoaudiology and Functional ENT Surgery "Prof. Dr. D. Hociota", Bucharest, Romania, ${ }^{2}$ University of Medicine and Pharmacy "Carol Davila", Bucharest, Romania, ${ }^{3}$ "Life-Med" Medical Center, Bucharest, Romania E-mail: ibulescu@gmail.com

\begin{abstract}
In the last decades, rhinology has developed in a great manner concerning new surgical techniques and extending the use of compatible biomaterials in order to achieve normal morphology and normal functional aspect after surgery (endoscopic or open approach). Since biomaterials are integrated in biological active organs and systems, they have to be easily accepted by the receiver with no toxicity, inflammation or other side effects. The authors try to review all biocompatible materials used in several rhinological pathologies in order to obtain the best results after functional or reconstructive surgery. In rhinological surgery there are usually used homograft's but the use of biomaterials like alloplastics (silicone, high density polyethylene, polytetrafluoroethylene, etc.) has widely increased. The authors present their experience in endoscopic approach of choanal atresia using nasal stents, closure techniques for septal perforation using nasal splints, endoscopic drainage and sinus ventilation using balloon sinuplasty, lacrimal pathway obstruction using double margin stent and some materials used in cosmetic and reconstructive rhinoplasty.
\end{abstract}

Keywords: biomaterials, implants, organic polymers, rhinologic surgery

\section{INTRODUCTION}

The use of compatible biomaterials has developed a lot in rhinologic pathology and reconstructive surgical techniques were described in order to obtain a functional result after surgery. Since biomaterials are integrated into biological organs and systems, it is desirable a technical functionality related to mechanical properties over time and an optimal biocompatibility, meaning limited inflammatory reaction, no toxicity or other side effects. The authors are proposing to review a wide variety of biomaterials currently available, along with their advantages and disadvantages for rhinological surgery. In addition to the constant use of autologous cartilage and homograft's, nowadays alloplastics are increasingly used. The rhinological conditions in which biomaterials can be applied are: choanal imperforation, chronic dacryocystitis, septal perforation, functional endoscopic surgery to maintain a patent paranasal sinus ostium, but also for reconstructive surgery.

\section{CHOANAL IMPERFORATION}

Choanal imperforation is a rare congenital condition in which the posterior region of nasal airflow is blocked by abnormal bony, membranous or fibrous tissue. In most cases, it's one-sided atresia, with less severe symptoms, detected at an older child or adult with possible associated malformations [1]. Surgery to remove the obstruction is an absolute indication. The endoscopic transnasal approach of unilateral choanal imperforation is the best choice, with minimal complications, fast recovery and increased success rate. At the end of the operation, placing a stent in a fixed position at the level of the choanal tunnel is highly recommended, for at least three months (Figure 1).

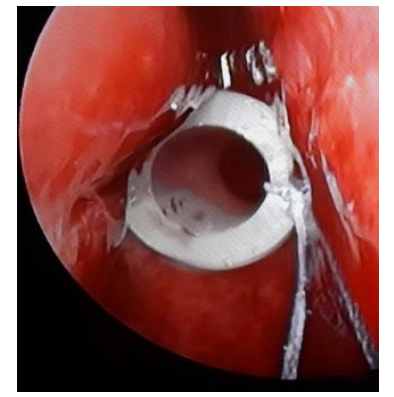

Figure 1. Intraoperative endoscopic appearance: the silicone stent fixed at the choanal

The tube should be made of soft material such as silicon or teflon to minimize the formation of granulation tissue (Figure 2). A good outcome involves avoidance of stenosis recurrence and depends on keeping the stent long enough but also on the quality of the material from which the tube is made.

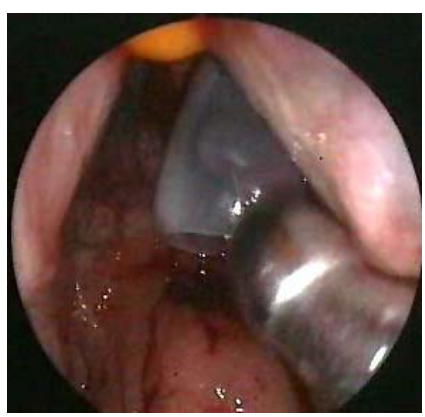

Figure 2. Intraoperative endoscopic view: the distal end of the stent reaching the rhinopharynx

\section{SEPTAL PERFORATION}

Septal perforation is an abnormal communication between the two nasal fossae which may have multiple causes: traumatic, infectious, neoplastic, degenerative, exposure to external and intranasal physical or chemical factors, like cocaine use, but may also be idiopathic. In the adult, the vast majority is iatrogenic, following septal 
deviation surgery. Perforations are classified according to their size and type, as well as location (front, middle and rear). These lesions especially the front ones may cause crusting, bleeding, whistling, pain, discharge and obstruction, but also nasal deformities (dorsal saddling or columellar retraction) [2]. Conservative treatment usually consists of emollients and humidification. Filling the perforation with a septal prosthesis or even surgical closing are available options for patients with persistent symptoms. Most of the subjects experienced an improvement or even a resolution of epistaxis, whistling and crusting after the procedure. Septal buttons have been made of acrylic and plastic in the past but are now primarily made of soft silicone (Silastic) (Figure 3 ).

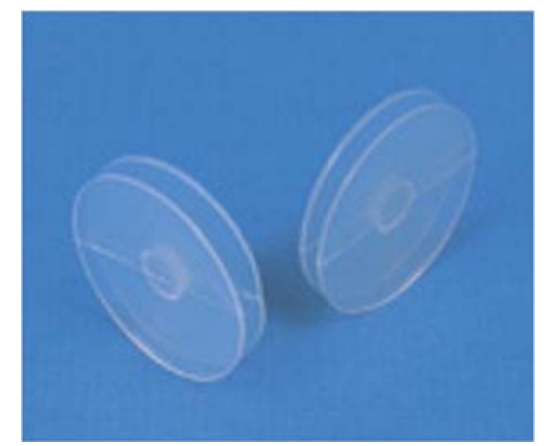

Figure 3. Nasal silicon septal button

\section{BALOON SINUPLASTY}

Balloon sinuplasty (BSP) represents the first step toward tissue preservation technique. Much like angioplasty [3], it uses a small, flexible balloon catheter for dilation of obstructed ostia at the level of the maxillary, sphenoid and frontal sinuses (Figure 4). BSP facilitates physiological drainage and ventilation with complete preservation of the native sinus anatomy and may be considered the treatment of choice for limited pathology. In patients with significant disease, BSP can be used as an adjunct to functional endoscopic sinus surgery.

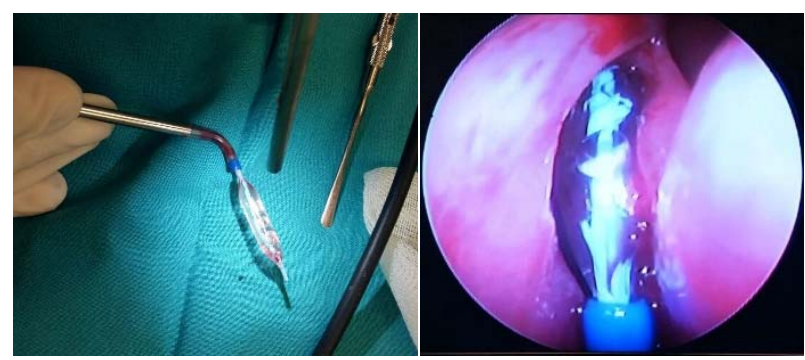

Figure 4. The balloon for sinuplasty, in the right frontal recess

\section{ENDOSCOPIC DACRYOCYSTORHINOSTOMY (DCR)}

The most common acquired causes for nasolacrimal duct obstruction include recurrent dacryocystitis, chronic inflammatory lacrimal diseases, radiotherapy and nasal/facial trauma, even iatrogenic lesions by repeated catheterization, septorhinoplasty and endoscopic sinus surgery.
Dacryocystorhinostomy is the standard treatment for saccal and postsaccal stenosis of the lacrimal apparatus. It represents a marsupialization of lacrimal sac inside the nasal cavity using an external or an endoscopic approach, with the aim of restoring tear drainage pathways. Neoostium cicatrisation followed by its closure is considered the main risk factor for surgical failure in endoscopic dacryocystorhinostomy. A wide neo-ostium together with insertion of a silicone stent may improve postoperative outcome of this technique [4]. We have developed a double margin tube, about 3-4 mm diameter, applied transnasally and positioned to the bony margin of the dacryocystorhinostoma. The stent is held in place for at least three months, resulting in the formation of a large fibrous ring, to ensure a long lasting permeability of the lacrimal pathways (Figure 5).

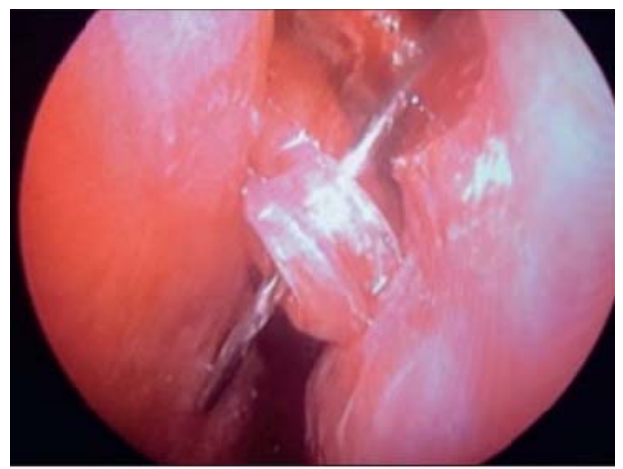

Figure 5. Endoscopic intraoperative view: double-margin silicon stent

\section{RHINOPLASTY}

Nasal reconstruction represents a significant challenge to the surgeon. Reestablishment of the aesthetic nasal contour and restoration of respiratory function are the dual goals of this effort. There are many grafting materials available, including autografts and homograft's and a wide variety of synthetic (alloplastic) materials (silicone, expanded polytetrafluoroethylene GORE-TEX and high density polyethylene) [5].

Silicone is widely used in rhinoplasty especially for the Asian patients. It can be inserted and also removed easily in case of complications. The main disadvantages of silicone use are: susceptibility to infection, columellar loss, encapsulation, calcification, extrusion or even rejection of the implant (Figure 6).
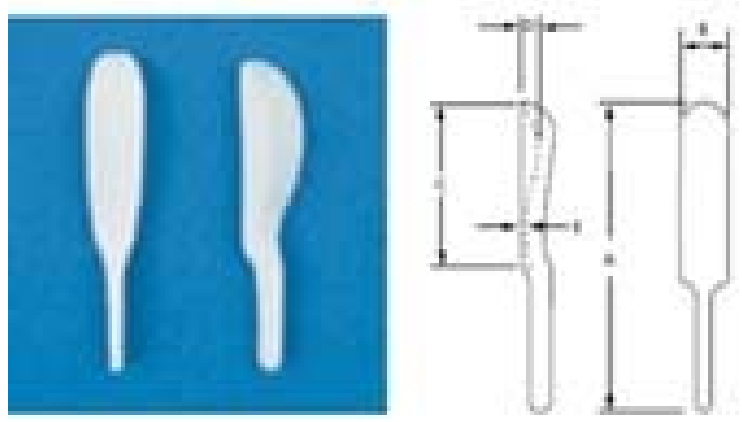

Figure 6. Silicone implant for dorsal augmentation 
Expanded polytetrafluoroethylene GORE-TEX has gained popularity for the augmentation rhinoplasty over the past years, with very small biological complication rate (inflammation and/or infection, extrusion). It is flexible and easy to use and has higher resistance to extreme temperatures than silicone. Its microporous nature enables in growth of soft tissue that provides adequate fixation, yet it allows its removal if necessary, but in a more complicated manner [6].

High density polyethylene has the greatest flexibility. It's also easy to handle. Implants let for fibro-vascular in growth which lends stability by offering a higher degree of cohesion with neighboring tissues, but makes the removal very difficult in case of complications (Figure 7).

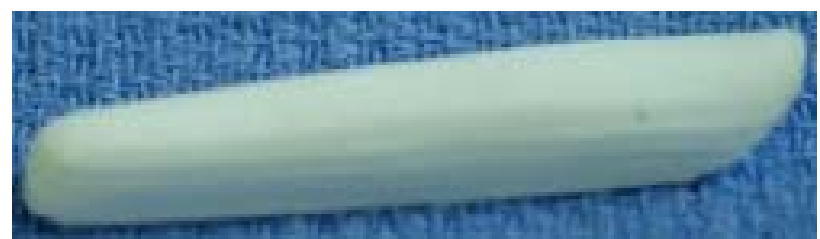

Figure 7. GORE-TEX material for nasal augumentation

\section{FACIAL RECONSTRUCTION SURGERY}

Surgical correction for large congenital or acquired craniofacial bone loss, including traumatic injuries or extensive tumour resections is another technical challenge. Biomaterials are used successfully during facial reconstruction to accomplish both cosmetic and functional sinonasal results. For example, after the complete removal of giant frontal sinus osteoma the anterior table must be simultaneously or secondarily reconstructed (Figure 8). In this situation Titanium plates ensure the greatest biocompatibility due to increased corrosion resistance as well as bone-like elastic profile. Also, this metal is characterized by great stability to a small mass [7].

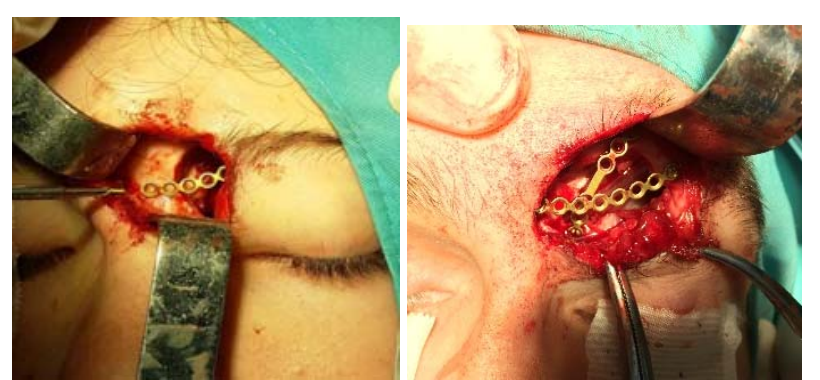

Figure 8. Intraoperative view: Titanium plate applied at the anterior wall of the frontal sinus

\section{CONCLUSIONS}

In the last decades, rhinology has evolved on a large scale. Technological progress along with the use of up-to-date biocompatible artificial substitutes have allowed the development of many endoscopic and open surgical procedures designed to achieve postoperative functional and aesthetic goals.

We are reiterating the concept of biocompatibility that means suitability with a living tissue or system by not being toxic, injurious or physiologically reactive and not causing immunological rejection [8].

Regardless of their temporary or permanent character, the biomaterials used in rhinologic surgery have to be easy to insert and must preserve the function of the organ they are applied in. The ideal implant material meets strict requirements concerning biocompatibility, plasticity, stability of form, resistance to infection and removability.

Last but not least the artificial substitutes problem should be considered from an economic point of view. The pricequality ratio is an important factor worth taking into account as health care coverage by the insurance companies as part of a public program.

\section{REFERENCES}

[1] Kwong, Kelvin M., Current Updates on Choanal Atresia, Frontiers in Pediatrics 3 (2015): 52. PMC. Web. 14 July 2017.

[2] Fernandez, Teny, Harshakumar Karunakaran, and Sheela Virginia Rodrigues, Prosthetic Management of a Nasal Septal Defect Using a Custom Made Unilateral Intranasal Stent: A Case Report, Journal of Clinical and Diagnostic Research: JCDR 10.8 (2016): ZD33-ZD34. PMC. Web. 14 July 2017.

[3] Ahmad, Zahoor, Balloon Sinuplasty, Indian Journal of Otolaryngology and Head \& Neck Surgery 62.3 (2010): 225-228. PMC. Web. 14 July 2017.

[4] Gauba, Vinod, External versus Endonasal Dacryocystorhinostomy in a Specialized Lacrimal Surgery Center, Saudi Journal of Ophthalmology 28.1 (2014): 36-39. PMC. Web. 14 July 2017.

[5] Kim, Hyun-Soo et al., Problems Associated with Alloplastic Materials in Rhinoplasty, Yonsei Medical Journal 55.6 (2014): 1617-1623. PMC. Web. 14 July 2017.

[6] Conrad K, Torgerson CS, Gillman GS., Applications of Gore-Tex implants in rhinoplasty reexamined after 17 years, Arch Facial Plast Surg. 2008 Jul-Aug. 10(4):224-31.

[7] Crosara, Paulo Fernando Tormin Borges et al., Rhinoplasty Complications and Reoperations: Systematic Review, International Archives of Otorhinolaryngology 21.1 (2017): 97-101. PMC. Web. 14 July 2017.

[8] Neumann, Andreas, and Kevin Kevenhoerster, Biomaterials for Craniofacial Reconstruction, GMS Current Topics in Otorhinolaryngology, Head and Neck Surgery 8 (2009): Doc08. PMC. Web. 13 July 2017. 